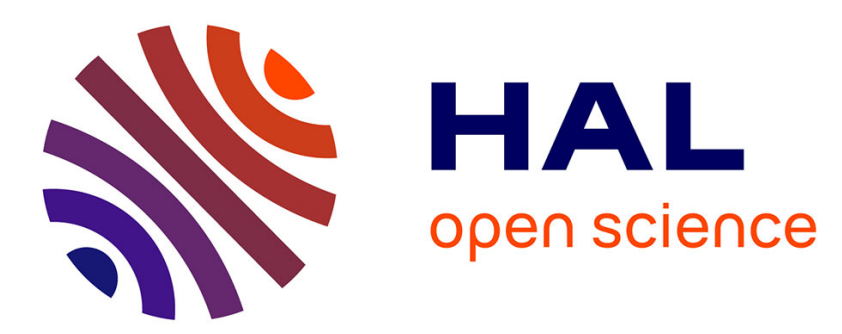

\title{
Enhancing Occupants Comfort and Well-being through a Smart Office setup
}

Ankica Barisic, Vasco Amaral, Moharram Challenger

\section{To cite this version:}

Ankica Barisic, Vasco Amaral, Moharram Challenger. Enhancing Occupants Comfort and Wellbeing through a Smart Office setup. 2020 43rd International Convention on Information, Communication and Electronic Technology (MIPRO), Sep 2020, Opatija, Croatia. pp.1825-1830, 10.23919/MIPRO48935.2020.9245212 . hal-03159933

\section{HAL Id: hal-03159933 https://hal.science/hal-03159933}

Submitted on 4 Mar 2021

HAL is a multi-disciplinary open access archive for the deposit and dissemination of scientific research documents, whether they are published or not. The documents may come from teaching and research institutions in France or abroad, or from public or private research centers.
L'archive ouverte pluridisciplinaire HAL, est destinée au dépôt et à la diffusion de documents scientifiques de niveau recherche, publiés ou non, émanant des établissements d'enseignement et de recherche français ou étrangers, des laboratoires publics ou privés. 


\title{
Enhancing Occupants Comfort and Well-being through a Smart Office setup
}

\author{
Ankica Barišić*‡, Vasco Amaral*, Moharram Challenger ${ }^{\dagger}$ \\ *Universidade NOVA de Lisboa, NOVA LINCS, DI, FCT, Lisboa, Portugal \\ ${ }^{\dagger}$ Electronics and ICT Department, University of Antwerp, Belgium \\ ${ }_{\ddagger}^{\ddagger}$ Center for Informatics and Computing Science, Ruđer Bošković Institute, Zagreb, Croatia \\ Email: a.barisic@campus.fct.unl.pt, vma@fct.unl.pt, moharram.challenger@uantwerpen.be
}

\begin{abstract}
A smart office, a particular case of Building Automation, represents a physical environment enriched with sensing, actuation, communication and computation capabilities aiming at acquiring and exploiting knowledge about the environment and its inhabitants. A fundamental goal of the smart offices is to ensure a comfortable working environment as well as adapting to the circumstances related to optimizing specific variables which indicate the safety and energy efficiency of the office, as well as the health and productivity of its occupants.

In this position paper, we illustrate a representative system by exploring emerging technologies, which presents a unique set of challenges and opportunities to research a new generation of software control systems, by supporting non-intrusive interfaces, that put the occupants at the center of the system's concerns, by focusing on comfort and well-being while still leveraging the occupants' behaviour.
\end{abstract}

\section{INTRODUCTION}

Smart buildings can be seen as a group of Cyber-Physical Systems (CPS) where the smart grid, the external communication networks with heterogeneous sensors and actuators, as well as its environment, interact by exchanging the energy and information while providing comfort services to humans. An effort is being made to establishing a reliable and sustainable technology for deploying green and zero-energy buildings [21]. Moreover, the buildings are being instrumented to automate and show intelligent behaviour.

While there are many projects and studies related to energy efficiency, the methods which consider assurance of the occupants' comfort and well-being are still in their early phase. The existing methods which take into consideration socio-technical aspects can be seen as too intrusive, as they ask the occupants for a constant input or feedback to adapt, which may be timeconsuming and disturbing. On the other hand, these methods often adopt the system based on post-occupancy data and predetermined parameters of energy exchanges, rather than acting on real-time situations and realistic sensing data (e.g. [20]). There are ample opportunities for improvements of efficient control through continuous evolution of real-life CPSs [9].

The current state-of-the-art and technology for building automation is a rule-based control which for instance use the current ambient temperature adjusted dynamically after every measurement to define set points [14]. Lack of this approach is that it is not able to dynamically respond to changing conditions, such as updates in temperature predictions, sunshine intensity or the assumptions on the number of occupants and their behaviour. Some researches suggest adopting predictive control model [12] which is supposed to better performance than the current control approach. Target expectations are better energy efficiency of the smart environment and enhancement of the occupant's comfort by incorporating the disturbance predictions (e.g. occupancy profiles, and changes in the outside environment) and deployment of distributed control procedures with adjustable parametrisation of the distributed agent-based controllers.

In the context of issues related to smart buildings, we focus on the smart office, which represents a more specific and realistic testing environment that we can control and where it is possible to isolate relevant parameters for experimentation. In the case of office setup, the occupant's primary goal is productivity related to their work tasks. Their productivity can be influenced by the number of factors, including their comfort, health and safety. The real-time feedback regarding the occupants can be collected through wearable and office sensors. Facility manager commonly provides new rules to a decision system which support the control of office behaviour by optimising the trade-offs between the energy savings and the occupant's comfort.

The paper is organised as follows: Section II summarises the regulations related to the offices, workplaces, and their occupants and workers. In Section III, the principles and terms used in smart offices are defined. The proposed smart office setup is detailed in Section IV. The challenges are discussed in Section V. Finally, the paper is concluded in Section VI.

\section{OCCUPATIONAL SAFETY AND HEALTH AT WORKPLACE}

According to the European Union (EU) regulations ${ }^{1}$, to protect the workers from work-related accidents and diseases, the European Commission has adopted EU Occupational Safety and Health (OSH) ${ }^{2}$ Strategic Framework 2014-2020 [10].

This framework identifies major challenges related to health and safety at work by calling for the implementation of existing health and safety rules and placing effective and efficient risk prevention strategies with tackling new and emerging risks without neglecting existing risks.

While well-being at work and working conditions have been improved by the introduction of new technologies and

\footnotetext{
${ }^{1}$ European Union Law: eur-lex.europa.eu

${ }^{2}$ OSH Wiki: oshwiki.eu
} 
innovations in work organisation, effective prevention of workrelated diseases requires anticipating potential adverse effects of new technologies on workers' health and safety. These technologies lead to new products and processes, which need to be sufficiently tested to ensure that they do not represent significant hazards for occupants and workers. Some of the emerging risks are linked to the development of biotechnologies and green technologies.

Changes in work organisations introduced by development of information technology, especially ones permitting constant connectivity, yield new flexible and interactive work processes solutions. According to the recent Euro-barometer survey reported in the OSH Strategic Framework, workers consider stress as one of the leading occupational risks (53\%), followed by ergonomic risks (repetitive movements or tiring or painful positions) (28\%) and lifting carrying or moving loads on a daily basis $(24 \%)$. This calls for specific attention to address the impact of changes in work organisation in terms of physical and mental health. One of the critical strategic objectives presented by the OSH Strategic Framework is improving statistical data collection to have better evidence and developing monitoring tools. Although, since 2013, the evidence-based policy is implemented to collect work-related accidents data, still remain to collect timely, reliable and comparable statistical data on occupational exposures and work-related ill-health, as well to analyse the costs and benefits in the area of OSH.

A risk assessment regarding OSH at work, as well implementation of prevention measures, must be performed by employers every time when a change is introduced in the workplace (e.g. new work equipment or procedure) or at least every year. The safety control measures to be implemented should be based on updated technical and/or organisational knowledge, and best practices. Prevention measures application aims to reduce the occupational disease occurrence and/or probability of work accident. It is expected that engineering or technical measures act directly on the risk source by removing the risks during the workplace design phase. Another way is to use administrative or organisational measures which promote proper attitudes and behaviours, as well as safety culture, by raising awareness through training, information sharing, and which implement appropriate working procedures and supervision, management and proactive monitoring, routine maintenance and housekeeping procedures and implementation of protection measures [10].

Employers are obligated to meet minimum requirements for workplaces predefined by EU commission. In a case of the smart office it is possible to automatise control over requirements referring to:

- Fire detection and fire fighting

- Ventilation of enclosed workplaces

- Room temperature

- Room lightning (natural and artificial)

- Windows and skylights

- Doors and gates

\section{Principles AND Methodology}

This section describes the terms used in this study for the proposed smart offices. Also, the general software and system techniques which can be used in the development of the proposed smart offices are defined. Finally, the main modules and high-level architecture are presented in this section.

The main perspectives of smart and sustainable office are:

- Well use of resources: Controlling the energy consumption by adapting to occupants presence, market trends and the outside environment, e.g. temperature, sunlight exposure, wind, humidity.

- Occupants Comfort: Supporting the occupants' work habits and health needs, e.g. considering if the person is unusually cold/warm, supporting notifications regarding drinking and eating needs, managing an appropriate light/noise level depending on the work tasks.

- Emergency and Resilience: Monitoring emergencies such as fire occurrence and gas leak [13], and help people to evacuate the building in the shortest time possible.

To address the real-time realisation of these perspectives, we suggest the following techniques to be exploited in the development of a smart office: modelling techniques, experimental software engineering, and dew computing.

Modelling techniques: Supporting the design and modelling of the system with domain-specific languages [6] and dataflow modelling [13] which can increase the level of abstraction and paves the way for tackling the complexity. Modelling techniques such as metamodelling [8] and generative languages [19][15] are used for the development of different IoT systems. Also, there is an example of smart office design and implementation by using a smart environmental metamodel as a framework for designing smart cyber-physical environments [5]. A model can present functional component interactions and offer and used services, along with a data meta-model that supplies the description of the properties and relationships of the data sources involved in the environment.

Experimental software engineering: To perform validation of design decisions or the evaluation of the smart office it is necessary to set up an experiment [4]. It is valuable to leverage sensors to capture indoor environmental quality or conditions in a work environment. To understand the occupant's variables such as comfort, satisfaction, health, and performance, it is essential to measure the environmental conditions across all variables and capture records of critical workplace attributes that define the physical environment. As an example, there is an integrated approach for building performance evaluation which match post-occupancy evaluation subjective tools by metrics [18], and is connected to database of over 1600 workstations with statistically significant findings about the measured and user-perceived quality of the indoor environment, as well as the technical attributes of building systems that contribute to successful, high performance buildings.

Dew computing: Using the transceiver devices the system can collect data [20] and identify the presence of the occupants [16] and their preferences with minimum disturbance e.g. by 


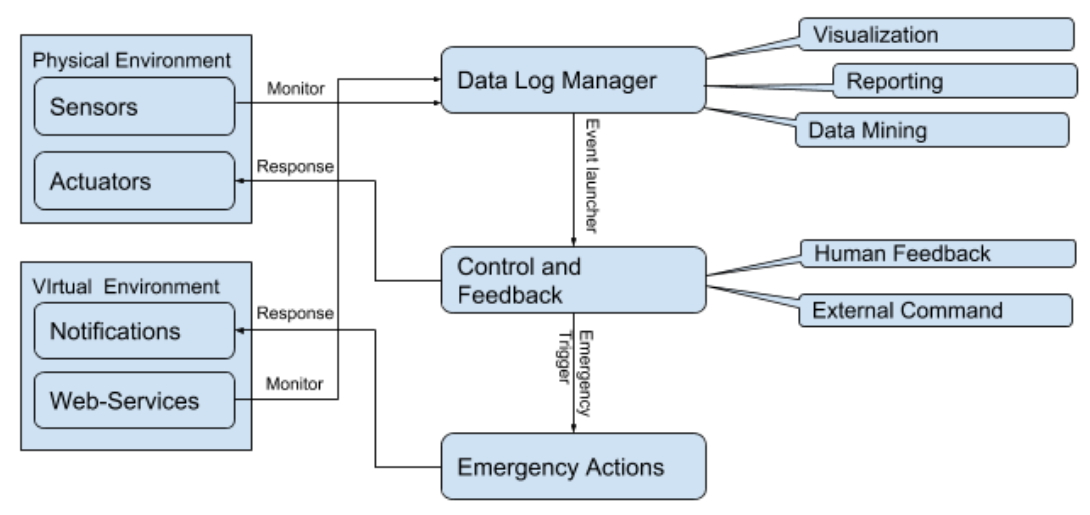

Fig. 1. The high-level architecture of a smart office

interpreting the needs based on the bio-signals instead of explicitly asking the human to control the office. Dew Computing paradigm is integrating the lowest level, physical-edge human environment-controlling devices into higher level behavioural systems. Tiny microcontrollers are in direct contact with the human physical environment, controlling a multitude of simple or just slightly complicated environmental processes. These are the micro-controllers in gas boilers, air-conditioning and heating equipments, solar power controllers, etc. These devices must possess self-sufficiency and can enable, by cooperating with devices in the same or higher level, interesting possibilities of energy saving, faster and more effective emergency service reaction and health-monitoring, among others. Dew devices, as micro-services, can be seamlessly integrated higher up into the computing hierarchy through Internet gateways into the Fog and Cloud, enabling the coordination of the physical edge control through common strategies, like adaptive-learning, predicting and cognitive computing [22]. For example, we can find the design specifications of an ECG mobile application which enables a real-time acquisition and processing using a given wearable bio-sensor [7].

Context awareness is a critical task for constructing the application of the smart systems and is mainly seen as the process that explicitly acquires the context from underlying sensing devices to support system performance. Suitable context representation model and reasoning approaches should be examined to enable the high-level semantic acquisition from the raw sensing data [3]. It is important to establishing continuous experimentation as a management instrument which result in continuous user satisfaction. In the area of CPSs, experimentation is underexplored and often not considered due to safety considerations and their proximity to the hardware level [11]. However, the CPS requirements are changing rapidly and therefore requiring a shift to iterative, humancentered development, in which continuous experimentation provides an excellent potential for facilitating its evolution and incrementally advancing its sustainability (economically, environmentally and in terms of usability). USE-ME framework [2] supports the design of empirical studies which take into consideration technical, physical and social elements by providing context-dependent metrics. This framework has the potential to model continuous experimentation of smart offices.

A smart office can be composed of three main groups of modules and components, including data collection and monitoring, control and actuation, and visualisation, reporting and data mining components.

Data collection and monitoring:

- It can collect the status of the office elements, e.g. whether windows/doors are open/closed, a light turned on/off, heating system, air conditioner, power sockets, office appliances (e.g. coffee machine, water heater) are working or not.

- It can retrieve the data from the outside environment, e.g. sunlight, temperature, air pollution, humidity, and wind speed.

- It can monitor the status of the occupant: presence, position, activity type, stress factors, body temperature, and existential needs (food, water, etc.).

Control and Actuation:

- To react to the environmental events, such as closing the windows/door, turning the light on/off, opening/closing the heating/cooling system.

- To notify or warn about the events, such as reminding to drink water or eat food, to warn about the difference of the temperature between the room and outside, and so on.

Visualisation, reporting, and data mining:

- Visualization and Reporting: The data can be visualised and reported in different forms to different people for analysis, building maintenance, and so on.

- The huge amount of the data (big data) collected from the office, the people and their behaviour during the time can be used for extracting meaningful data and finding anomalies, patterns and correlations to predict the future events and behaviours.

These modules and components can be used in a smart office by adopting the architecture presented in Figure 1. The system includes the log manager, control and feedback, and physical and virtual environments. The log manager collects 


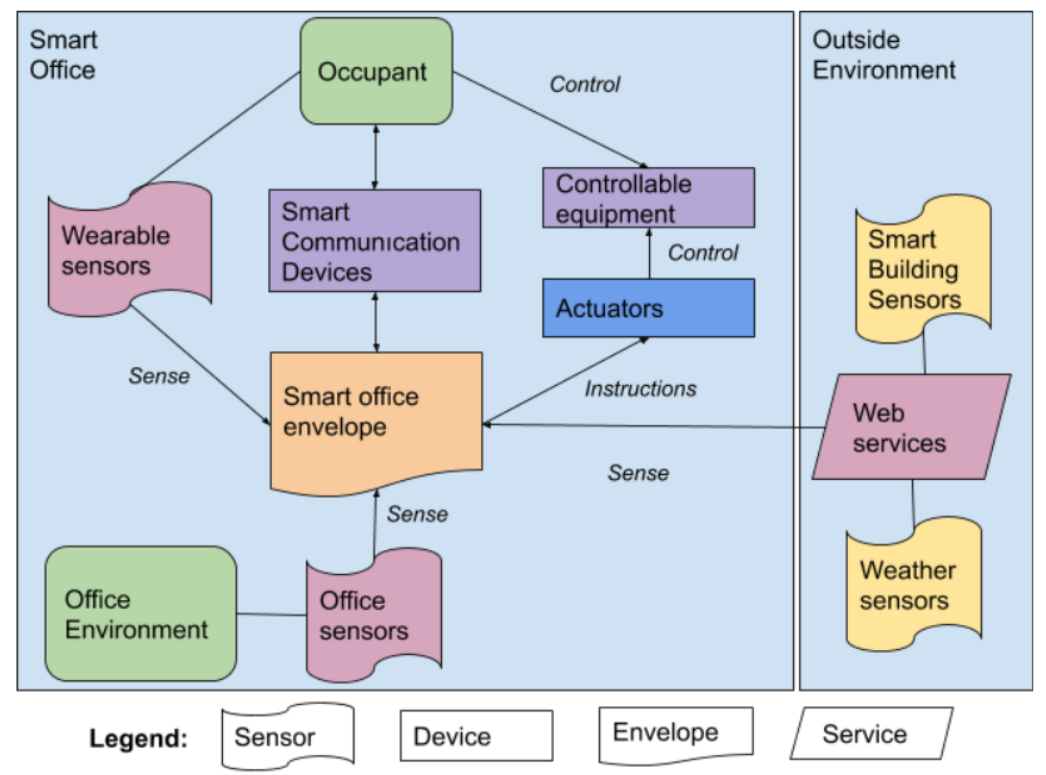

Fig. 2. The smart Office Setup model

the data and provides the events according to the pre-defined rules. These events can be used in the control and feedback module to send instructions to the actuators in the physical environment and/or messages as notifications to the users' smart devices such as a smartphone in the virtual environment. These are considered as the responses to the events. The control section can detect the emergencies, such as fire occurrence or gas leak [1][14], and can trigger related actions in the system.

The system collects data with sensors in the physical environment and forms the web-services in the virtual environment. It can use this processed information to visualise them (for each of the users), report (for management issues), and do data mining (for example, to find anomalies). Finally, the system can receive the feedback of the user or instructions from admins.

The system can support run-time features such as control of the office equipment, occupants health and safety conditions, and energy consumption. Also, it can support design-time features such as the support for facility manager (planning for resource management).

\section{Smart Office Setup}

The smart office, discussed in this paper, is representing a common work-place, not the one which requires special conditions. It is not, for example, a work-place with no air or light. The central part of the office is a Smart Office Envelope (SOE) which performs control and sensing actions over the Inside Environment, Outside Environment and the Occupants (see Figure 2).

SOE via integration of smart technologies is an autonomic system to respond to the environment alterations and user behaviour. It controls the technical services like heating, ventilation, air-conditioning, lights, electrical power, and network. The occupants and technical services are placed in spatial sequence (interior walls, desk, chair, work equipment) of Inside Environment [17].

The system supports interaction between the virtual model and the physical model. The Occupants can communicate with SOE by Smart Communication Devices (such as a smartphone, office computer, and smart-watch) to provide a control input or feedback, as well as to receive a notification from the SOE. Occupants play a proactive role in the smart office, not just as part of the inside environment, but also considering the sensors/actuators.

Each occupant has manual control over the Controllable Equipments in the office, such as windows, doors, lights, heating, air conditioning, ventilation, etc. The SOE can control this equipment through the set of instructions based on the rules which take the real-time data to make a decision. The SOE control over the equipment, in most of the normal conditions, should not override the actions of the occupants. On the other hand, the decision can be made based on the data which is collected from:

- Occupants - through the Wearable Sensors (e.g. biosensors and trackers) from which it is possible to obtain information regarding their body temperature, if they are hot/warm, hungry/thirsty, and in some cases measure a level of stress indicators.

- Office Environment - through the Office Sensors, such as object detector, face recognition system, light sensor, and so on, which can capture the information such as the level of light, noise, temperature, wind, humidity, air pollution (in specific part of the office), and the position of the occupant.

- Outside Environment - through the Web Services which obtain the information from the web-based sources such as Weather Sensors (forecast) and Smart Building Data. For example, the Weather Sensors can provide information regarding the level of daily light, temperature, wind, 
humidity and air pollution in the outside environment. Also, Smart Building Data can give information such as emergency events in the building in which the office is located.

It is worth to note that the Weather Sensors can be in most cases found in the outside environment infrastructure, but if the service which provides this information is not available, it is possible to replace the outside sensors with the office infrastructure (e.g. from the outside part of the windows).

Inside Environment reacts to the occupant's behaviour and the Outside Environment. The system's objective is to optimise the occupant's comfort, use of energy (such as electricity consumption), as well as hazard and risk prevention, while the occupant's goal is the execution of the work assignments - as they have tasks, schedules, and they use tools and equipment.

To make the application of the system clear, here we present a representative scenario for a smart office. Let's consider a laboratory with some researchers working in it. Upon the occupant's agreement, they wear some bio-sensors, let say in the form of a wrist-watch or a necklace, to collect some biological information such as heart bit rate, body temperature, blood pressure, breathing rate and so on. On the other hand, the office can have sensors for temperature, humidity, $\mathrm{CO} 2$, smoke, and so on. The data can be stored in a database and the control can be done using a data-flow model, using dataflow programming such as NodeRed. This control mechanism can access the building data, such as catastrophic events and announcement. Also, the system is integrated with outside services such as weather forecast system. This control part of the system can handle the environmental equipment such as windows, doors, air condition, light, and so on, using some actuators and control devices such as step motors, remote switches, and so on.

This setup can pave the way to realise the requirements discussed in the previous sections mainly focusing on the occupant's comfort, energy saving, and hazard/risk management. In the realisation of these features, a golden rule is not to disturb the occupants and avoid unnecessary work interruptions. For example, the system can perceive the tiredness, thirstiness, or hungriness of the occupants and give some suggestions and recommendations based on the available facilities recorded in the system, to prevent the unhealthy situation, which will impact the performance of the people. This can be also considered for the stressful times of the occupants by checking the body information and analysing the person's schedule and come up with useful suggestions, e.g. having a short walk or grabbing some food.

Also, the system can remind the staff when to leave the office, considering the air-pollution, the traffic, the appointments which he/she may have, and/or, as facilitator, by coordinating with some other colleague who plan to leave at the same time and close vicinity, to go together, resulting less pollution, safer, and healthier transportation. The system even can consider the temperature difference between the office, the building, and outside and based on this input can automatically close the window and open the air-condition. For instance, in a high level of air pollution at the outside of the building, a system can consume more energy in favour of the health of the occupants, without annoying them by control instructions.

Finally, the system can detect hazardous events, such as fire ignition, gas leak, and so on, at the earliest time possible and give an alarm and guide the occupants to evacuate the office at the earliest time possible. In this regard, the system can consider the exits, the number of people in each office and immediately direct the people in a way that avoid unnecessary rush and crowd, which usually lead to bottlenecks and even injuries/casualties.

\section{Challenges}

Successful implementation of the discussed office setup implies the following major challenges to be tackled:

Enhanced decision support systems - which balance rational use of resources, occupants comfort and emergency and resilience. Many works are addressing a rational use of resources by providing the occupants with information on how to reduce their energy spending. However, there are not many approaches which focus on ensuring occupants safety and comfort. We need accurate models that would allow us to reliably predict and simulate the behaviour of the work office which could be standardised.

User-friendly tools - with multi-modal user interfaces supporting the different stakeholders such as office designers, occupants and facility managers to facilitate appropriate humancomputing interaction. For the design phase, we need the tools to help the system's prescription. Also, during the commissioning period and the system's run-time, the occupants should be supported with the tools that enable easy feedback processing and individual control. Besides, facility managers, induced by tools that present the controllers' monitoring input, stream data analysis (with learning patterns), and the legal framework should be able to use the feedback to trigger a change in the setup of the office appliances infrastructure, rules and also behaviour. For that, easy to use tools and languages are needed.

Feedback data collection - of smart office may not only interact with humans (occupants and facility managers) but also communicate with the other systems using network infrastructures (e.g. outside environment and smart building). Collecting data from the occupants' through wearable sensors should be seen as a complementary source of information resulting in the office environment improvements by adopting it to the individual needs. However, the office should be resilient by operating even when this data is not available by relying on the office sensors.

Continuous experimentation - rely on the data continuously collected by smart office system and is a powerful resource for activities such as run-time diagnosis, early problem identification, improvement of system functionality, and delivery of the new features and products. However, its successful application complicates the organisational processes as different stakeholders ( e.g. engineers, developers, and managers) should be coupled with each other. Finally, it is necessary to have supportive instruments for experimentation 
which provide virtual test environments for the new updates and extensions or configuration changes of the office system.

Conformance with legal aspect - is an essential aspect while addressing statistical data collection. Given the variety of situations in terms of company size and diversity of the workforce, the interpretation of the legal issues can be ambiguous and should be often updated. However, while there is a need to design targeted and effective policy measures, OSH strategic framework calls for non-legislative tools to be used (i.e. benchmarks, identifying and exchanging good practices, awareness-raising, among others). Several EU funds are created to support the design and implementation of innovative and more productive ways of the workplace with the objective to increase coherence between environmental policy and worker's protection.

\section{CONCLUSions AND Future Work}

In this position paper, we present a smart office setup which exploits the new-age possibilities to improve the monitoring and control of the smart office while ensuring occupants' comfort, well use of resources and the emergency and resilience perspectives. There is a lack of effective settings and tools which support the design and analysis phases of smart-offices. They can be addressed by exploiting the emerging technologies (i.e. modelling techniques, and experimental software engineering and dew computing techniques).

The proposed office setup collects the data continuously through the office and wearable sensors. Although the impact of wearable sensors is still an under-exploration, the data continuously collected from these sensors can contribute to a systematic and highly automated virtual testing.

As a future work, we plan to adopt the approach in the context of Smart Office called SmartLab which is being implemented in our laboratory. The idea is to develop adaptive mechanisms which can automatically incorporate the measured information into the prediction models.

\section{ACKNOWLEDGMENT}

This research was (partly) supported by: i) the Scientific and Technological Research Council of Turkey (TUBITAK) under grant 115E591, and ii) Portuguese grants NOVA LINCS Research Laboratory (Grant: FCT/MCTES PEst UID/ CEC/04516/2013) and DSML4MAS Project (Grant: FCT/MCTES TUBITAK/0008/2014), and (iii) the European Regional Development Fund under the grant KK.01.1.1.01.0009 (DATACROSS).

\section{REFERENCES}

[1] S. Arslan, M. Challenger, and O. Dagdeviren. Wireless sensor network based fire detection system for libraries. In 2nd International Conference on Computer Science and Engineering, UBMK 2017, 2017.

[2] A. Barišić, V. Amaral, and M. Goulão. Usability Driven DSL development with USE-ME. Computer Languages, Systems and Structures (ComLan), 51:118-157, 2018.

[3] A. Barišić, D. Blouin, V. Amaral, and M. Goulão. A Requirements Engineering Approach for Usability-Driven DSL Development. In 10th International Conference on Software Language Engineering (SLE), Vancouver, British Columbia, Canada, 2017. ACM.
[4] A. Barišić, C. Debreceni, D. Varro, V. Amaral, and M. Goulao. Evaluating the efficiency of using a search-based automated model merge technique. In 2018 IEEE Symposium on Visual Languages and HumanCentric Computing (VL/HCC), pages 193-197. IEEE, oct 2018.

[5] F. Cicirelli, G. Fortino, A. Guerrieri, G. Spezzano, A. Vinci, F. Cicirellia, G. Fortino, A. Guerrieri, G. Spezzano, and A. Vinci. Metamodeling of Smart Environments: from design to implementation. Advanced Engineering Informatics, 33:274-284, 82017.

[6] S. Demirkol, M. Challenger, S. Getir, T. Kosar, G. Kardas, and M. Mernik. Sea_l: A domain-specific language for semantic web enabled multi-agent systems. In 2012 Federated Conference on Computer Science and Information Systems, FedCSIS 2012, pages 1373-1380. IEEE, 2012.

[7] E. Domazet, M. Gusev, and L. Antovski. Design specification of an ECG mobile application. In 2017 25th Telecommunication Forum (TELFOR), pages 1-4. IEEE, 112017.

[8] C. Durmaz, M. Challenger, O. Dagdeviren, and G. Kardas. Modelling contiki-based IoT systems. In OpenAccess Series in Informatics, volume 56, 2017.

[9] S. Engell, R. Paulen, C. Sonntag, H. Thompson, M. Reniers, S. Klessova, and B. Copigneaux. Proposal of a European Research and Innovation Agenda on Cyber-physical Systems of Systems - 2016-2025. www.cpsos.eu, 2016.

[10] C. European. EU Occupational Safety and Health (OSH) Strategic Framework 2014-2020. Technical report, Brussels, 2014.

[11] F. Giaimo, H. Yin, C. Berger, and I. Crnkovic. Continuous Experimentation on Cyber-Physical Systems. In Proceedings of the Scientific Workshop Proceedings of XP2016 on - XP '16 Workshops, pages 1-2, New York, New York, USA, 2016. ACM Press.

[12] T. Johnson, A. Kerzhner, C. J. J. Paredis, and R. Burkhart. Integrating Models and Simulations of Continuous Dynamics Into SysML. Journal of Computing and Information Science in Engineering, 12(1):011002, 3 2012.

[13] B. Karaduman, T. Asici, M. Challenger, and R. Eslampanah. A Cloud and Contiki based Fire Detection System using Multi-Hop Wireless Sensor Networks. In Proceedings of the Fourth International Conference on Engineering \& MIS 2018, page 66. ACM, 2018.

[14] B. Karaduman, M. Challenger, and R. Eslampanah. ContikiOS based library fire detection system. In 2018 5th International Conference on Electrical and Electronic Engineering (ICEEE), pages 247-251. IEEE, 2018.

[15] G. Kardas, B. T. Tezel, and M. Challenger. Domain-specific modelling language for belief-desire-intention software agents. IET Software, 12(4):356-364, 2018.

[16] N. Karimpour, B. Karaduman, A. Ural, M. Challenger, and O. Degdeviren. IoT based Hand Hygiene Compliance Monitoring. In The 2019 International Symposium on Networks, Computers and Communications (ISNCC'19), Istanbul, 2019. IEEE (To be Published).

[17] D.-Y. Kim and S.-A. Kim. An exploratory model on the usability of a prototyping- for designing of Smart Building Envelopes. Automation in Construction, 81:389-400, 92017.

[18] V. Loftness, V. Hartkopf, A. Aziz, J.-H. Choi, and J. Park. Critical Frameworks for Building Evaluation: User Satisfaction, Environmental Measurements and the Technical Attributes of Building Systems (POE + M). In Building Performance Evaluation, pages 29-48. Springer International Publishing, Cham, 2018.

[19] H. M. Marah, R. Eslampanah, and M. Challenger. DSML4TinyOS: Code Generation for Wireless Devices. In 2nd International Workshop on Model-Driven Engineering for the Internet-of-Things (MDE4IoT), 21st International Conference on Model Driven Engineering Languages and Systems (MODELS2018), Copenhagen, Denmark, 2018.

[20] L. Özgür, V. K. Akram, M. Challenger, and O. Dagdeviren. An IoT based smart thermostat. In 2018 5th International Conference on Electrical and Electronic Engineering (ICEEE), pages 252-256. IEEE, 2018.

[21] P. H. Shaikh, N. B. M. Nor, P. Nallagownden, I. Elamvazuthi, and T. Ibrahim. A review on optimized control systems for building energy and comfort management of smart sustainable buildings. Renewable and Sustainable Energy Reviews, 34:409-429, 62014.

[22] Z. Sojaat and K. Skala. The dawn of Dew: Dew Computing for advanced living environment. In 2017 40th International Convention on Information and Communication Technology, Electronics and Microelectronics (MIPRO), pages 347-352. IEEE, 52017. 